\title{
Structural basis of FbpA-mediated periplasmic iron transport in Moraxella catarrhalis
}

Clement Chan ckochan@ucalgary.ca

Dixon Ng dixon.ng@ucalgary.ca

MeghaShah megha.shah@utoronto.ca

Nobu Watanabe nobuhiko.watanabe@ucalgary.ca

Christine Lai chiehlin.lai@utoronto.ca

Alexei Savchenko alexei.savchenko@ucalgary.ca

Trevor Moraes trevor.moraes@utoronto.ca

Anthony Schryvers schryver@ucalgary.ca

Moraxella catarrhalis is an opportunistic pathogen that can cause disease in

immunocompromised individuals and manifest in existing conditions, such as upper respiratory tract infection or otitis media. Conventional use of antibiotics has become increasingly less effective due to the emergence of antimicrobial resistant strains. The development of a crossprotective vaccine could provide a long-term solution to resistant strains and confer protection for immune vulnerable individuals. M. catarrhalis transferrin and lactoferrin receptors are proteins that are suitable for vaccine development, due to their important role in helping to maintain bacterial iron homeostasis during colonization and pathogenesis. The periplasmic transport of ferric iron mediated by FbpABC in $\mathrm{M}$. catarrhalis has not been studied. My research focuses the structural basis of FbpA-mediated iron transport through the periplasm. We report a $1.83 \AA \AA$ apoFbpA structure and $1.86 \AA ̊$ structure of FbpA bound to an iron nanocluster. 